\title{
Inverse Optimization Method of Resource Allocation with Consideration of Advantage Structure
}

\author{
Lili Zhang ${ }^{1,2}$, Wenwen Yang ${ }^{1} \&$ Dejun Teng ${ }^{3}$ \\ ${ }^{1}$ School of Business, Dalian University of Technology, Dalian, China \\ ${ }^{2}$ Dalian University of Technology-Beijing Research Institute, Beijing, China \\ ${ }^{3}$ Anshan Iron \& Steel Group Quality Test Center, Anshan, China \\ Correspondence: Lili Zhang, School of Business, Dalian University of Technology, Dalian 116024, China.
}

Received: February 7, 2017

Accepted:February 22, 2017

Online Published: March 20, 2017

doi:10.5430/ijba.v8n 2p86

URL: https://doi.org/10.5430/ijba.v8n2p86

Fund by: National natural science foundation of China (71401023); Fund by humanities and social science of education ministry China (14YJC630191); Fund of Social science development in liaoning province (2017lslktyb-049); Fund of Social science development in Dalian city(2016dlskyb013).

\begin{abstract}
Abs tract
The research collects competence items of informal questionnaire by expert interview method, analyzes the results qualitatively and quantitatively and forms formal questionnaire. Through statistical analysis and AMOS modeling, the research obtains workers' competence model and validates competence model. An identification method of individual advantage characters according competency indicator system is built up relying on programming analysis and parameter optimization. We build the optimization model and its inverse optimization model, starting from the original optimization model, adjusting parameter value as small as possible, the conventional optimization model translated into the inverse model by the principle of duality. A calculation example is used to make sure the method is reliability and feasibility.

Keywords: competence model, advantage structure model, inverse optimization model, inverse resources allocation

\section{Introduction}

Competency plays an important role to promote the development of human resources performance, identifying individual advantage structure is vital to ensure person-job match scientific, advantage structure is identified by means of weight vector in optimization model, determined by making as small distance between evaluation value vector and ideal value vector as possible. MBO (Management by Objective), under the goal setting theory, based on objective management, for a given optimization problem, the inverse optimization problem is to find a minimal adjustment of the parameters (costs, capacities, etc.) of the problem such that the given solutions become optimum. Inverse optimization problem is used extensively in resources allocation, it is very useful for goal management which means when the goal is set, the resources adjustment can be done according to the goal and for the minimu m change of the original model, and the classical numerical algorithm and intelligent algorithm for the inverse optimization problem have been developed.
\end{abstract}

\section{Competency Model Empirical Research}

The concept of "competency" was first proposed in 1970s. It is the personal condition and behavior characteristics of the outstanding person and the ordinary person in a certain field, such as motivation, knowledge, attitude, trait and so on. With the development of society, the concept of "competency" is more and more concerned and valued (Rodriguez, D., Patel, R., Bright, A., et al., 2002, pp.309-324). Before qualitatively and quantitatively analysis, the AMOS structural equation model has 142 variables, 69 observable variables, 73 unobserved variables, 73 exogenous variables, 69 endogenous variable. The competency path model is showed in Table 1. 
Table 1. Path coefficient of regression analys is

\begin{tabular}{|c|c|c|c|c|c|c|c|c|c|c|c|c|c|c|c|c|c|}
\hline & & & & & C.R. & & Label & & & & & & & C.R. & & Label & \\
\hline a1 & $<---$ & $\mathrm{F} 1$ & 1.000 & & & & & .696 & b15 & $<---$ & $\mathrm{F} 2$ & .249 & .029 & 8.570 & $* * *$ & par_34 & .757 \\
\hline $\mathrm{a} 2$ & $<---$ & $\mathrm{F} 1$ & .825 & .069 & 12.031 & $* * *$ & par_1 & .690 & b14 & $<---$ & $\mathrm{F} 2$ & .269 & .031 & 8.613 & $* * *$ & par_35 & .766 \\
\hline a3 & $<---$ & $\mathrm{F} 1$ & .819 & .067 & 12.256 & $* * *$ & par_2 & .703 & b13 & $<---$ & $\mathrm{F} 2$ & .270 & .031 & 8.636 & $* * *$ & par_36 & .771 \\
\hline $\mathrm{a} 4$ & $<---$ & $\mathrm{F} 1$ & .752 & .064 & 11.666 & $* * *$ & par_3 & .669 & b12 & $<---$ & $\mathrm{F} 2$ & .273 & .032 & 8.532 & $* * *$ & par_37 & .749 \\
\hline a5 & $<---$ & $\mathrm{F} 1$ & 1.067 & .075 & 14.211 & $* * *$ & par_4 & .823 & b11 & $<---$ & $\mathrm{F} 2$ & .297 & .034 & 8.708 & $* * *$ & par_38 & .786 \\
\hline a6 & $<---$ & $\mathrm{F} 1$ & 1.079 & .078 & 13.889 & $* * *$ & par_5 & .804 & b10 & $<---$ & $\mathrm{F} 2$ & .282 & .032 & 8.690 & $* * *$ & par_39 & .782 \\
\hline a7 & $<---$ & $\mathrm{F} 1$ & .951 & .074 & 12.925 & $* * *$ & par_6 & .746 & b9 & $<---$ & $\mathrm{F} 2$ & .284 & .033 & 8.720 & $* * *$ & par_40 & .788 \\
\hline a8 & $<---$ & $\mathrm{F} 1$ & .902 & .069 & 13.013 & $* * *$ & par_7 & .752 & $\mathrm{~b} 8$ & $<---$ & $\mathrm{F} 2$ & .265 & .031 & 8.571 & $* * *$ & par_41 & .756 \\
\hline a9 & $<---$ & $\mathrm{F} 1$ & 1.128 & .080 & 14.070 & $* * *$ & par_8 & .814 & b7 & $<---$ & $\mathrm{F} 2$ & .293 & .034 & 8.669 & $* * *$ & par_42 & .777 \\
\hline a10 & $<---$ & $\mathrm{F} 1$ & 1.036 & .077 & 13.380 & $* * *$ & par_9 & .773 & b6 & $<---$ & $\mathrm{F} 2$ & .266 & .031 & 8.609 & $* * *$ & par_43 & .764 \\
\hline a11 & $<---$ & $\mathrm{F} 1$ & 1.079 & .075 & 14.324 & $* * *$ & par_10 & .829 & b5 & $<---$ & $\mathrm{F} 2$ & .260 & .030 & 8.567 & $* * *$ & par_44 & .755 \\
\hline a12 & $<---$ & $\mathrm{F} 1$ & 1.004 & .074 & 13.560 & $* * *$ & par_11 & .784 & b4 & $<---$ & $\mathrm{F} 2$ & .300 & .035 & 8.553 & $* * *$ & par_45 & .752 \\
\hline a13 & $<---$ & $\mathrm{F} 1$ & 1.106 & .082 & 13.421 & $* * *$ & par_12 & .775 & b3 & $<---$ & $\mathrm{F} 2$ & .304 & .035 & 8.689 & $* * *$ & par_46 & .781 \\
\hline a14 & $<---$ & $\mathrm{F} 1$ & .965 & .073 & 13.244 & $* * *$ & par_13 & .765 & $\mathrm{~b} 2$ & $<---$ & $\mathrm{F} 2$ & .333 & .038 & 8.857 & $* * *$ & par_47 & .820 \\
\hline a15 & $<---$ & $\mathrm{F} 1$ & .970 & .071 & 13.704 & $* * *$ & par_14 & .792 & b1 & $<---$ & $\mathrm{F} 2$ & .330 & .038 & 8.781 & $* * *$ & par_48 & .802 \\
\hline a16 & $<---$ & $\mathrm{F} 1$ & .994 & .072 & 13.841 & $* * *$ & par_15 & .801 & $\mathrm{c} 1$ & $<---$ & F3 & 1.000 & & & & & .645 \\
\hline a17 & $<---$ & $\mathrm{F} 1$ & .906 & .066 & 13.633 & $* * *$ & par_16 & .788 & $\mathrm{c} 2$ & $<---$ & F3 & 1.497 & .124 & 12.035 & $* * *$ & par_49 & .775 \\
\hline a18 & $<---$ & $\mathrm{F} 1$ & .931 & .070 & 13.323 & $* * *$ & par_17 & .770 & c3 & $<---$ & F3 & 1.279 & .112 & 11.406 & $* * *$ & par_50 & .725 \\
\hline a19 & $<---$ & $\mathrm{F} 1$ & 1.003 & .073 & 13.660 & $* * *$ & par_18 & .790 & $\mathrm{c} 4$ & $<---$ & F3 & 1.115 & .101 & 11.005 & $* * *$ & par_51 & .695 \\
\hline a20 & $<---$ & $\mathrm{F} 1$ & .995 & .070 & 14.149 & $* * *$ & par_19 & .819 & $c 5$ & $<---$ & F3 & 1.630 & .129 & 12.651 & $* * *$ & par_52 & .826 \\
\hline a21 & $<---$ & $\mathrm{F} 1$ & .762 & .061 & 12.528 & $* * *$ & par_20 & .722 & c6 & $<---$ & F3 & 1.684 & .132 & 12.722 & $* * *$ & par_53 & .832 \\
\hline $\mathrm{a} 22$ & $<---$ & $\mathrm{F} 1$ & .929 & .072 & 12.935 & $* * *$ & par_21 & .747 & $\mathrm{c} 7$ & $<---$ & F3 & 1.572 & .124 & 12.665 & $* * *$ & par_54 & .827 \\
\hline a23 & $<---$ & $\mathrm{F} 1$ & .887 & .069 & 12.910 & $* * *$ & par_22 & .745 & $\mathrm{c} 8$ & $<---$ & F3 & 1.499 & .125 & 12.025 & $* * *$ & par_55 & .774 \\
\hline a24 & $<---$ & $\mathrm{F} 1$ & .984 & .069 & 14.188 & $* * *$ & par_23 & .821 & c9 & $<---$ & F3 & 1.440 & .117 & 12.294 & $* * *$ & par_56 & .796 \\
\hline a25 & $<---$ & $\mathrm{F} 1$ & .933 & .067 & 14.006 & $* * *$ & par_24 & .810 & $\mathrm{c} 10$ & $<---$ & F3 & 1.459 & .119 & 12.276 & $* * *$ & par_57 & .795 \\
\hline a26 & $<---$ & $\mathrm{F} 1$ & .898 & .065 & 13.859 & $* * *$ & par_25 & .802 & c11 & $<---$ & F3 & 1.233 & .108 & 11.396 & $* * *$ & par_58 & .725 \\
\hline a27 & $<---$ & $\mathrm{F} 1$ & 1.051 & .074 & 14.227 & $* * *$ & par_26 & .823 & $\mathrm{c} 12$ & $<---$ & F3 & 1.089 & .102 & 10.697 & $* * *$ & par_59 & .672 \\
\hline a28 & $<---$ & $\mathrm{F} 1$ & 1.004 & .073 & 13.843 & $* * *$ & par_27 & .800 & c13 & $<---$ & F3 & 1.606 & .123 & 13.022 & $* * *$ & par_60 & .857 \\
\hline a29 & $<---$ & $\mathrm{F} 1$ & .952 & .070 & 13.580 & $* * *$ & par_28 & .784 & $\mathrm{c} 14$ & $<---$ & F3 & 1.444 & .118 & 12.256 & $* * *$ & par_61 & .793 \\
\hline a30 & $<---$ & $\mathrm{F} 1$ & .977 & .069 & 14.075 & $* * *$ & par_29 & .814 & c15 & $<---$ & F3 & 1.045 & .097 & 10.733 & $* * *$ & par_62 & .674 \\
\hline a31 & $<---$ & $\mathrm{F} 1$ & 1.015 & .075 & 13.600 & $* * *$ & par_30 & .786 & d1 & $<---$ & $\mathrm{F} 4$ & 1.000 & & & & & .876 \\
\hline a32 & $<---$ & $\mathrm{F} 1$ & 1.050 & .076 & 13.901 & $* * *$ & par_31 & .804 & $\mathrm{~d} 2$ & $<---$ & $\mathrm{F} 4$ & 1.006 & .044 & 23.080 & $* * *$ & par_63 & .898 \\
\hline a33 & $<---$ & $\mathrm{F} 1$ & .954 & .070 & 13.581 & $* * *$ & par_32 & .784 & $\mathrm{~d} 3$ & $<---$ & $\mathrm{F} 4$ & 1.030 & .043 & 23.971 & $* * *$ & par_64 & .914 \\
\hline \multirow[t]{2}{*}{ a34 } & $<---$ & $\mathrm{F} 1$ & .938 & .069 & 13.504 & $* * *$ & par_33 & .780 & $\mathrm{~d} 4$ & $<---$ & $\mathrm{F} 4$ & .971 & .046 & 21.220 & $* * *$ & par_65 & .863 \\
\hline & & & & & & & & & d5 & $<---$ & F4 & .839 & .045 & 18.768 & $* * *$ & par_66 & .809 \\
\hline
\end{tabular}


The correlation coefficient between the latent variables of competency in structural model is shown in Table 2 .

Table 2. The correlation coefficient between the latent variables

\begin{tabular}{|c|c|c|c|c|c|c|c|c|}
\hline & & & covariance & S.E. & C.R. & $P$ & Label & coefficient \\
\hline $\begin{array}{l}\text { successful general } \\
\text { characteristics }\end{array}$ & $\langle--\rangle$ & $\begin{array}{c}\text { knowledge of automatic } \\
\text { production line }\end{array}$ & 1.000 & & & & & .834 \\
\hline risk control ability & $\langle-->$ & $\begin{array}{l}\text { successful general } \\
\text { characteristics }\end{array}$ & .290 & .034 & 8.645 & $* * *$ & par_67 & .749 \\
\hline $\begin{array}{l}\text { successful general } \\
\text { characteristics }\end{array}$ & $\langle--\rangle$ & professional quality & .187 & .024 & 7.905 & $* * *$ & par_68 & .820 \\
\hline $\begin{array}{l}\text { knowledge of } \\
\text { automatic } \\
\text { production line }\end{array}$ & $<-->$ & professional quality & .574 & .057 & 9.998 & $* * *$ & par_69 & .800 \\
\hline risk control ability & $<-->$ & $\begin{array}{c}\text { knowledge of automatic } \\
\text { production line }\end{array}$ & .777 & .071 & 10.941 & $* * *$ & par_70 & .637 \\
\hline risk control ability & $\langle-->$ & professional quality & .157 & .020 & 7.859 & $* * *$ & par_71 & .676 \\
\hline
\end{tabular}

Fitting degree evaluation if competency in structuralmodel is shown in Table 3.

Table 3. Fit index of competency structural model

\begin{tabular}{|c|c|c|c|c|c|c|c|c|c|c|c|c|}
\hline Model & NFI & RFI & IFI & TLI & CFI & $\begin{array}{l}\text { RMS } \\
\text { EA }\end{array}$ & $\begin{array}{l}\text { PCLO } \\
\text { SE }\end{array}$ & $\begin{array}{l}\text { NPA } \\
\mathrm{R}\end{array}$ & CMIN & $\mathrm{DF}$ & $\mathrm{P}$ & $\begin{array}{l}\text { CMIN/ } \\
\text { DF }\end{array}$ \\
\hline default & .626 & .602 & .680 & .658 & .678 & .097 & .000 & 213 & 10623.321 & 2271 & 0 & 4.678 \\
\hline saturated & 1.0 & & 1.00 & & 1.0 & .167 & .000 & 2484 & .000 & 0 & & \\
\hline independent & .0 & .000 & .000 & .000 & .000 & & & 69 & 28389.69 & 2415 & 0 & 11.756 \\
\hline
\end{tabular}

To sum up, the competency indicator system contains the following indicators: knowledge of automatic production line, successfulgeneral characteristics, profes sional quality.

\section{Advantage Structure Identification Model}

In fact, evaluations always have guiding functions, which usually contains three major aspects: the first one is determined by each indicator of evaluation index system. The second one is determined by the result of the ideal value. This function is only in object programming evaluation methods. The third one is expressed by the vector of value parameter, the vector of value parameter could be used to express their advantages and weaknesses.

The advantage structure identification model should be comparable to the ideal condition which provides a clear guidance in the behavior adjusting process. Since the limitation of detection technology, level of many indexes can not be evaluated straightly (such as competency). In this case, using collected information, which can be obtained by questionnaire through choosing a number of respondents who have contacted with the evaluated object, inviting them to assess object's behavior level on each ite $\mathrm{m}$. Since one respondent can not acquaint in all respects, it needs to get the evaluation information by group decision making. Although valuations are usually influenced by certain environment with a psychological tendency to express one's talent and public image. Th is tendency moves valuations to cover up his emotional preferences and display "justness". In other words, even if the existence of subjective influence on the evaluation such as psychological effect, fairly reveal the object's strength and weakness. So this paper uses average score as indicator value.

Moreover, each decision maker has his unique angle which we can hardly judge whether it is right or wrong then and there, and the evaluation object hopes to be evaluated by maximization its social contribution or work efforts and obtained achievements from standing out their individual advantage characteristics. So it is necessary to find out the 
object's quantitative advantage structure based on observers'evaluation, and get one aggregate-value.

Among the existing utility functions, the object oriented utility function is object programming evaluations. There are $p$ indicators, Person $i$ 's advantage structure identification model is:

$$
d^{2}\left(x_{i}-x^{*}\right)=\sum_{j=1}^{p} w_{j}^{2}\left(x_{j}^{*}-x_{i j}\right)^{2}
$$

where $x^{*}=\left(x_{1}^{*}, x_{2}^{*}, \cdots, x_{p}^{*}\right)^{\tau}$ represents the ideal value of each indicator, $x_{i}=\left(x_{i 1}, x_{i 2}, \cdots, x_{i p}\right)^{\tau}$ refers to respondent evaluation value on the object of each indicator, $w=\left(w_{1}, w_{2}, \cdots, w_{p}\right)^{\tau}$ is the vector of value parameter (weight vector). Actually, function (1) gives the distance (square) between the evaluation value and ideal value.

In order to identify object's individual advantage structure from the point of view of each respondent, to determine the vector weight by making the object most propitious. It can be carried out by model (2) (Zhang, L., Hou, X., \& Xi, F., 2013, pp.160-164):

$$
\begin{gathered}
\min d_{i}^{2}\left(x_{i}, x^{*}\right)=\sum_{j=1}^{p} w_{i j}^{2}\left(x_{j}^{*}-x_{i j}\right)^{2} \\
\text { s.t }: \quad \sum_{j=1}^{p} w_{i j}=1 \\
w_{i j} \geq 0 \quad j=1,2, \cdots p ; i=1,2, \cdots n
\end{gathered}
$$

The objective function in model (2) is continuous, and the feasible region of constrained conditions is bounded, so the most optimal solutions of model (2) exist. The solution set of function(2) is as following:(i)If there is at least one evaluation value equal to ideal value, then the sum of the components which reach the ideal value is 1 , other index weights are all 0; (ii)If there is no evaluation value reaching ideal value, the weight can be obtained by (Zhang, L., 2011, pp.102-105):

$$
\begin{gathered}
w_{i j}^{*}=\frac{\lambda_{i}^{*}}{\left(x_{i j}^{*}-x_{i j}\right)^{2}} \\
\lambda_{i}^{*}=\frac{1}{\sum_{j=1}^{p} \frac{1}{\left(x_{i j}^{*}-x_{i j}\right)^{2}}}, i=1,2, . ., n, j=1,2, \ldots, p
\end{gathered}
$$

The comprehensive evaluation method is:

$$
c_{i}=\sum_{j=1}^{p} w_{i j} x_{i j}
$$

\section{Optimization Model and Inverse Optimization Model}

The optimization model is as following.

$$
\begin{aligned}
& \max (\min ) f=\sum_{j=1}^{p} w_{1 j} x_{1 j} y_{1}+\sum_{j=1}^{p} w_{2 j} x_{2 j} y_{2}+\cdots+\sum_{j=1}^{p} w_{n j} x_{n j} y_{n} \\
& \text { s.t. }\left\{\begin{array}{l}
a_{11} y_{1}+a_{12} y_{2}+\cdots+a_{1 n} y_{n} \leq(=, \geq)=b_{1} \\
a_{21} y_{1}+a_{22} y_{2}+\cdots+a_{2 n} y_{n} \leq(=, \geq)=b_{2} \\
\cdots \\
a_{m 1} y_{1}+a_{m 2} y_{2}+\cdots+a_{m n} y_{n} \leq(=, \geq)=b_{m} \\
y_{j} \geq 0(j=1,2, \ldots, n)
\end{array}\right.
\end{aligned}
$$


When seeing an optimization problem, we usually assume that the parameters such as costs, capacities, efficiency etc., are known and that we are interested in finding an optimal solution. However, in practice, it may happen that we only know estimates for the para meters. Additionally, we might know that certain are optimal from observations. The idea of inverse optimization is to find values of the parameters which make the known solutions optimum and which differ from the given estimates as little as possible (Heuberger, C., 2004, pp.329-361).

Inverse optimization based on the linear optimization model. Making a linear programming model of a given solution to realize optimization by adjusting the coefficient vector. According to the feasible solution of the optimal solution, the optimal solution of the plan can be achieved by adjusting the cost coefficient or the resource constraint. The method based on linear inverse problem enables companies to be more flexible in corresponding demand.

The optimization model is:

$$
\begin{aligned}
& \min z=c^{T} y \\
& \text { (s.t.) }\left\{\begin{array}{l}
A y \geq b \\
y \geq 0
\end{array}\right.
\end{aligned}
$$

A kind of dual problems of linear programming is established as the following.

$$
\begin{aligned}
& \max \mathrm{z}=\omega b \\
& \text { (s.t.) }\left\{\begin{array}{l}
\omega A \leq c \\
\omega \geq 0
\end{array}\right.
\end{aligned}
$$

Given model (7) a feasible solution $y^{0}$, make the inverse optimization conversion, make $y^{0}$ the optimal solution, according to the dual theory, model (8) has the optimal solution $\omega^{0}$. Then:

$$
\begin{gathered}
\omega^{0}\left(A y^{0}-b\right)=0 \\
\left(c-\omega^{0} A\right) y^{0}=0 \\
\min \|\bar{c}-c\| \\
\text { s.t. }\left\{\begin{array}{l}
\omega^{0} a_{j} \leq \overline{c_{j}}, y_{j}=0 \\
\omega^{0} a_{j}=\overline{c_{j}}, y_{j}>0
\end{array}\right.
\end{gathered}
$$

$\overline{c_{j}}=c_{j}+\varepsilon_{j}-\beta_{j}$, total cost change is: $\varepsilon-\beta$, the inverse optimization can be changed as:

$$
\begin{aligned}
& \min \|\varepsilon+\beta\| \\
& \text { s.t. }\left\{\begin{array}{l}
\omega^{0} a_{j}-\varepsilon_{j}+\beta_{j} \leq c_{j}, y_{j}=0 \\
\omega^{0} a_{j}-\varepsilon_{j}+\beta_{j}=c_{j}, y_{j}>0 \\
\varepsilon_{j}, \beta_{j} \geq 0
\end{array}\right.
\end{aligned}
$$


Given model (7) a non-feasible solution $y^{0}$, make the inverse optimization conversion, make $y^{0}$ the optimal solution, according to the dual theory, model (8) has the optimal solution $\omega^{0}$. Then:

$$
\begin{aligned}
& \min \|\bar{b}-b\| \\
& \text { s.t. }\left\{\begin{array}{l}
a_{i} y^{0}-b_{i} \geq 0, \omega_{i}=0 \\
a_{i} y^{0}-b_{i}=0, \omega_{i}>0
\end{array}\right.
\end{aligned}
$$

Let $\overline{b_{i}}=b_{i}+\alpha_{i}-\theta_{i}, \mathrm{i}=1,2, \ldots, \mathrm{m}, \alpha_{i} \geq 0, \theta_{i} \geq 0$, then:

$$
\begin{aligned}
& \min \|\alpha+\beta\| \\
& \text { s.t. }\left\{\begin{array}{l}
a_{i} y^{0}-\alpha_{i}+\beta_{i} \geq b_{i}, \omega_{i}=0 \\
a_{i} y^{0}-\alpha_{i}+\beta_{i}=b_{i}, \omega_{i}=0 \\
\alpha_{i} \geq 0 \\
\beta_{i} \geq 0
\end{array}\right.
\end{aligned}
$$

\section{Calculation Example Analysis}

$$
\begin{aligned}
& \operatorname{minz}=15 x_{1}+24 x_{2}+5 x_{3} \\
& (\text { s.t. })\left\{\begin{array}{l}
6 x_{2}+x_{3} \geq 2 \\
5 x_{1}+2 x_{2}+x_{3} \geq 1 \\
x_{1}, x_{2}, x_{3} \geq 0
\end{array}\right.
\end{aligned}
$$

Applying simplex algorithm, the optimal solution of the optimization problem is $y^{0}=(0,1 / 4,1 / 2)$.

We want to make a non-feasible solution $x^{0}=(1 / 5,1 / 5,0)$ the optimal solution for the model( 7$)$, we need to change $b$, so the mathematical model is:

$$
\begin{aligned}
& \min u=\alpha_{2}+\beta_{2}+\alpha_{1}+\beta_{1} \\
& \alpha_{1}-\beta_{1} \geq \frac{4}{5} \\
& \text { (s.t.) }\left\{\begin{array}{l}
\alpha_{2}-\beta_{2} \geq-\frac{2}{5} \\
\alpha_{1}, \beta_{1}, \alpha_{2}, \beta_{2} \geq 0
\end{array}\right.
\end{aligned}
$$


We use MATLAB to solve this problem, the optimal solution is $(0,0.4,0,0)$, and we change $b_{1}$ from 2 to 1.6 , others do not need to change.

\section{Conclusion}

The arrival of intelligent era will not only intelligent applied to all areas of life, but also for future workers put forward a severe demand. In the future, especially in the industries with high intelligence penetration, people need to evaluate their work with their own new ideas. Because some indicators measure inconvenience, the details of the division of competencies are built. Based on the existing performance evaluation methods, this paper gives a method of identifying the advantage structure of individuals.

This paper incorporates the academic thinking of inverse optimization, not only puts psychology and behavior into optimization model, but also data mines strengths characteristics under the psychology and behavior data, and find a new way to introducing the strengths characteristics into optimization model.

\section{References}

Heuberger, C. (2004). Inverse comb inatorial optimization: a survey on problems, methods, and results. Journal of Combinatorial Optimization, 8(3), 329-361. https://doi.org/10.1023/B:JOCO.0000038914.26975.9b

Liu, Y. (2012). Inverse Linear programming by modifying the right hand sight vector. A Thesis Submitted to Southeast University For the Academic Degree of Master of Operations Research and Cybernetics.

Rodriguez, D., Patel, R., Bright, A., et al. (2002). Developing competency models to promote integrated human resource practices. Human Resource Management, 41(3), 309-324. https://doi.org/10.1002/hrm.10043

Zhang, L. (2011). Group identification method for features of human capital inner quality structure. Journal of Management and Strategy, 2(3), 102-105. https://doi.org/10.5430/jms.v2n3p102

Zhang, L., Hou, X., \& Xi, F. (2013). Research on entrepreneur environment management evaluation method derived from advantage structure. Research Journal of Applied Sciences, Engineering and Technology, 6(1), 160-164. 